\title{
Variabilidade espacial do intervalo hídrico ótimo de solos cultivados em sistema plantio direto
}

\author{
Vilson Antonio Klein ${ }^{(1)}$, Gilvan Jaciel Graebin ${ }^{(2)}$, Delcio Rudinei Bortolanza ${ }^{(1)}$ e André Guilherme Daubermann ${ }^{(1)}$ \\ (1)Universidade de Passo Fundo, Laboratório de Física e Água do Solo, BR 285, São José, CEP 99052-900 Passo Fundo, RS, Brasil. \\ E-mail: vaklein@upf.br, delcio.bortolanza@outlook.com, agdguilherme@gmail.com ${ }^{(2)}$ Faculdade Concórdia, Rua Anita Garibaldi, no 3.185, \\ Primavera, CEP 89701-130 Concórdia, SC, Brasil. E-mail: gilvangraebin@gmail.com
}

Resumo - O objetivo deste trabalho foi avaliar a acurácia das regressões que definem os limites do intervalo hídrico ótimo e a validade do uso deste indicador ao se considerar a variabilidade espacial de lavouras sob sistema plantio direto. Foram coletadas 100 amostras de solo com estrutura preservada, em áreas com dimensões de 10x10 m, em Latossolo Vermelho distrófico, Nitossolo Vermelho distroférrico e Latossolo Vermelho aluminoférrico, manejados sob sistema plantio direto. Determinaram-se porosidade de aeração $\left(0,10 \mathrm{~m}^{3} \mathrm{~m}^{-3}\right)$, capacidade de campo (potencial de $-0,006 \mathrm{MPa}$ ), ponto de murcha permanente (potencial de $-1,5 \mathrm{MPa})$ e resistência mecânica do solo à penetração crítica $(2 \mathrm{MPa})$. As regressões que descreveram a capacidade de campo tiveram os menores coeficientes de determinação, enquanto as que descreveram a porosidade de aeração, o ponto de murcha permanente e a resistência mecânica do solo à penetração apresentaram os maiores valores. As regressões que definem o limite da capacidade de campo não são acuradas em descrever a variação na umidade volumétrica do solo por meio da densidade do solo, e os solos avaliados apresentam elevada variabilidade espacial para o intervalo hídrico ótimo.

Termos para indexação: capacidade de campo, ponto de murcha permanente, porosidade de aeração, resistência mecânica do solo à penetração.

\section{Spatial variability of the least limiting water range of soils under a no-tillage system}

\begin{abstract}
The objective of this work was to evaluate the regressions that define the least limiting water range limits and the validity of using this indicator considering the spatial variability of crops under a no-tillage system. One hundred undisturbed soil samples were collected in areas with dimensions of 10x10 m, in two different Oxisols and an Ultisol managed under no-tillage. Air-filled porosity $\left(0.10 \mathrm{~m}^{3} \mathrm{~m}^{-3}\right)$, field capacity (potential of $-0.006 \mathrm{MPa}$ ), permanent wilting point (potential of $-1.5 \mathrm{MPa}$ ), and soil mechanical resistance ( $2 \mathrm{MPa}$ ) were determined. The regressions that described field capacity had the lowest coefficients of determination, whereas those that described air-filled porosity, permanent wilting point, and soil mechanical resistance to penetration showed the highest values. Regression adjustments that define field capacity limits are not accurate in explaining variation on volumetric soil water content through bulk density, and the evaluated soils show high spatial variability regarding the least limiting water range.
\end{abstract}

Index terms: field capacity, permanent wilting point, air-filled porosity, soil mechanical resistance to penetration.

\section{Introdução}

O intervalo hídrico ótimo (IHO), amplamente utilizado como indicador da qualidade física e estrutural do solo (Silva et al., 1994; Lapen et al., 2004; Leão et al., 2006; Silva et al., 2006), é definido por uma faixa de conteúdo de água do solo, na qual são mínimas ou ausentes as limitações ao crescimento das plantas, ocasionadas por indisponibilidade de água, deficiência de aeração e resistência do solo à penetração (Silva et al.,1994; Kaiser et al., 2009; Safadoust et al., 2014). O IHO permite quantificar os mecanismos e os processos de perda e recuperação da qualidade física do solo, em função do seu uso e manejo (Guimarães et al., 2013), e geralmente é adotado para relacionar propriedades do solo e práticas de manejo (Safadoust et al., 2014).

Há diversos relatos da utilidade do IHO como indicador da qualidade física de diferentes tipos de solos, culturas e manejos (Silva et al., 1994, 2004; 
Betz et al., 1998; Tormena et al., 1999; Wu et al., 2003; Lapen et al., 2004; Leão et al., 2006; Neményi et al., 2006). Beutler et al. (2007), por exemplo, avaliaram a compactação, um dos processos de perda da qualidade do solo que afeta a produtividade de plantas, e verificaram que o IHO foi eficiente no monitoramento desse fator em cultivo de soja de sequeiro e irrigado. Já Betioli Júnior et al. (2012), ao analisar solo após 30 anos de plantio direto, constataram diminuição do IHO em função do aumento da densidade do solo, independentemente do limite crítico de resistência à penetração (2 $\mathrm{MPa})$, embora, acima deste limite, o IHO tenha sido condizente com a qualidade física do solo.

Contudo, apesar de bastante utilizado, o IHO ainda não foi consolidado no meio científico como bom indicador da qualidade do solo, e, portanto, sua validade tem sido colocada à prova (Gubiani et al., 2013b; Jong van Lier \& Gubiani, 2015; Cecagno et al., 2016). Jong van Lier \& Gubiani (2015) não recomendaram seu uso como indicador da qualidade física do solo, pois não abrangeu condições de contorno fenológicas e pedológicas comuns, e atribuíram sua frequência de uso a sua facilidade de obtenção. Esses mesmos autores ainda ressaltaram que os limites do IHO são estabelecidos em parâmetros e relações empíricas, não baseados em modelos físico-químicos dos processos de transferência de massa e energia no sistema solo-planta-atmosfera. Cecagno et al. (2016) também concluíram que o IHO não é um indicador adequado da qualidade física do solo em sistemas de integração lavoura-pecuária, uma vez que não apresentou nenhuma relação direta com o rendimento de grãos de soja. Segundo estes autores, indicadores fisiológicos podem representar de forma mais eficiente a interação entre o sistema solo-planta-atmosfera e o tráfego de máquinas e animais.

Embora o IHO seja afetado pela variabilidade espacial do solo, ainda são poucas as pesquisas sobre o assunto (Cavalcante et al., 2011). Em áreas agrícolas, além da variabilidade natural, são acrescentadas fontes adicionais de heterogeneidade no solo devido ao manejo (Camargo et al., 2010). O IHO obtido, então, é bastante diferenciado dentro de uma mesma classe de solo, com mesmo manejo. Além disso, muitas vezes, os ajustes das funções que definem o IHO são baixos ou não são apresentados.
Nesse sentido, faz-se necessário o estudo do comportamento das propriedades físicas do solo, bem como de modelos matemáticos que as descrevam em função da variabilidade espacial, para que mais evidências suportem ou não a utilização e/ou a eficiência do IHO.

O objetivo deste trabalho foi avaliar a acurácia das regressões que definem os limites do intervalo hídrico ótimo e a validade do uso deste indicador ao se considerar a variabilidade espacial de lavouras sob sistema plantio direto.

\section{Material e Métodos}

O experimento foi realizado no Rio Grande do Sul em: Latossolo Vermelho distrófico $\left(\mathrm{L}_{480}\right)$, no Município de Passo Fundo; em Nitossolo Vermelho distroférrico $\left(\mathrm{N}_{520}\right)$, no Município de Pontão; e em Latossolo Vermelho aluminoférrico $\left(\mathrm{L}_{640}\right)$, no Município de Tapejara (Streck et al., 2008). A composição granulométrica dos solos está descrita na Tabela 1 . Todos os solos foram manejados sob sistema plantio direto por mais de dez anos, sem revolvimento, com dois cultivos anuais: milho (Zea mays L.) ou soja [Glycine max (L.) Merr.] no verão; e trigo (Triticum aestivum L.) ou aveia (Avena sativa L.) no inverno.

Em cada sítio amostral, foram coletadas 100 amostras de solo, com estrutura preservada, por meio de cilindros volumétricos de $100 \mathrm{~cm}^{3}$ acoplados a um trado coletor, na camada de 2,5 a $7,5 \mathrm{~cm}$. As amostras foram coletadas em 2014, em área com dimensões de 10x10 m, espaçadas entre si em $1 \mathrm{~m}$ nos sentidos transversal e paralelo.

A densidade do solo (DS) foi obtida por meio da relação entre a massa de solo seco da amostra e o seu volume ocupado no cilindro (Blake \& Hartge, 1986). A umidade volumétrica do solo $(\theta)$ para porosidade de aeração foi determinada ao se subtrair $0,10 \mathrm{~m} \mathrm{~m}^{-3} \mathrm{da}$ porosidade total. Já a porosidade total foi calculada pela subtração do volume total do cilindro do volume ocupado pelos sólidos do solo, o qual foi obtido a partir da relação entre a DS e a densidade de partículas, determinada pelo método do balão volumétrico (Claessen, 1997).

A umidade na condição de capacidade de campo (CC) foi assumida no potencial $(\psi)$ de $-0,006 \mathrm{MPa}$ e obtida em funis de placa porosa (Klein, 2014). A umidade gravimétrica no ponto de murcha

Pesq. agropec. bras., Brasília, v.51, n.11, p.1890-1898, nov. 2016 DOI: $10.1590 / \mathrm{S} 0100-204 \mathrm{X} 2016001100011$ 
permanente (PMP) foi determinada no $\psi$ de $-1,5 \mathrm{MPa}$, em psicrômetro modelo WP4-T (Decagon Devices, Pullman, WA, EUA), seguido-se as instruções do fabricante, e convertida em $\theta$ com base na DS.

A resistência mecânica do solo à penetração (RP) foi determinada em laboratório, por penetrógrafo computadorizado, obtendo-se a leitura da força aplicada em intervalos de $1 \mathrm{~s}$, na velocidade constante de penetração de $0,17 \mathrm{~mm} \mathrm{~s}^{-1}$, com cone de $4 \mathrm{~mm}$ de diâmetro da base (área da base $=12,56 \mathrm{~mm}^{2}$ ) e semiângulo de $30^{\circ}$. As amostras foram ensaiadas, uma única vez, em diferentes umidades, determinadas ao se secar a amostra em estufa a $105^{\circ} \mathrm{C}$. A média dos 200 valores centrais do cilindro constituiu a RP medida da amostra. A RP foi ajustada em função da DS e da $\theta$, por meio da seguinte equação (Busscher, 1990): RP = $\mathrm{a} \times \mathrm{DS}^{\mathrm{b}} \times \theta^{\mathrm{c}}$

Esta equação foi rearranjada para obter a $\theta$ na qual é atingida a RP limitante de $2 \mathrm{MPa}$, tendo-se utilizado o algoritmo sugerido por Leão et al. (2005):

$$
\theta_{\mathrm{RP}}=\left(2 / \mathrm{a} \times \mathrm{DS}^{\mathrm{b}}\right)^{\frac{1}{\mathrm{c}}}
$$

Nas equações, RP é a resistência mecânica do solo à penetração $(\mathrm{MPa}) ; \theta$ é a umidade volumétrica $\left(\mathrm{m}^{3} \mathrm{~m}^{-3}\right)$; DS é a densidade do solo $\left(\mathrm{Mg} \mathrm{m}^{-3}\right) ; \theta_{\mathrm{RP}}$ é a umidade na qual a RP torna-se crítica (2 MPa); e a, b, e c são os parâmetros empíricos de ajuste, determinados iterativamente no ajuste dos dados ao modelo.

Os resíduos de todas as regressões utilizadas foram transformados em escores padronizados (z-score), e as amostras cujo valor foi superior ou inferior a três foram consideradas discrepantes (outliers) e eliminadas da análise (Hair Junior et al., 2009). Os dados obtidos de porosidade de aeração, CC e PMP foram linearizados em função da DS. Já a $\theta_{R P}$ foi descrita em função da DS por meio da equação rearranjada. Para a determinação dos coeficientes da primeira equação, utilizou-se o

Tabela 1. Composição granulométrica dos solos avaliados.

\begin{tabular}{|c|c|c|c|c|}
\hline Classe de solo & $\begin{array}{c}\text { Abrevia- } \\
\text { tura }\end{array}$ & Argila & $\begin{array}{l}\text { Silte } \\
-\left(\mathrm{g} \mathrm{kg}^{-1}\right)\end{array}$ & Areia \\
\hline Latossolo Vermelho distrófico & $\mathrm{L}_{480}$ & 480 & 160 & 360 \\
\hline Nitossolo Vermelho distroférrico & $\mathrm{N}_{520}$ & 520 & 130 & 350 \\
\hline Latossolo Vermelho aluminoférrico & $\mathrm{L}_{640}$ & 640 & 300 & 60 \\
\hline
\end{tabular}

Pesq. agropec. bras., Brasília, v.51, n.11, p.1890-1898, nov. 2016 DOI: 10.1590/S0100-204X2016001100011 método dos mínimos quadrados e o algoritmo de Levenberg-Marquardt.

\section{Resultados e Discussão}

Os coeficientes obtidos para a função de Busscher (1990) estão representados na Tabela 2, e o ajuste da função, pelo teste $F$, foi significativo para os três solos avaliados $(\mathrm{p}<0,001)$. Os coeficientes de determinação foram de 0,68, 0,80 e 0,70 para $\mathrm{L}_{480}, \mathrm{~N}_{520}$ e $\mathrm{L}_{640}$, respectivamente (Figura 1). Assim, grande parte da variação na RP foi explicada em função da DS e da $\theta$. Apesar disso, quando se analisam os valores de RP medidos e estimados pelo ajuste da função de Busscher (1990), nota-se um desvio considerável da linha de referência 1:1 (Figura 2). A regressão linear que define os dados estimados em função dos obtidos, tem como coeficiente angular os valores de 0,5705, 0,7844 e 0,6875 para $\mathrm{L}_{480}, \mathrm{~N}_{520}$ e $\mathrm{L}_{640}$, respectivamente, sendo o valor desejável 1 . O coeficiente linear da regressão é igual a $0,8805,0,6727$ e 0,7672 para $\mathrm{L}_{480}$, $\mathrm{N}_{520}$ e $\mathrm{L}_{640}$, respectivamente, sendo o valor desejável 0 . Como consequência, a primeira equação superestimou os menores valores de RP e subestimou os maiores. Entretanto, apesar dessa distorção, quando avaliado o valor de interesse, ou seja, RP $=2 \mathrm{MPa}$, os valores obtidos e estimados ficaram próximos entre si.

Normalmente, nos trabalhos em que se determinam o IHO, assume-se o valor da densidade de partículas de 2,65 $\mathrm{g} \mathrm{cm}^{-3}$ (Leao et al., 2005; Guedes Filho et al., 2013; Safadoust et al., 2014), e, desse modo, a variação na porosidade de aeração depende exclusivamente da variação na DS. Contudo, não há embasamento teórico que justifique a utilização dos valores utilizados recorrentemente apenas com base em outros trabalhos em que foram adotados (Jong van Lier \& Gubiani, 2015). No presente trabalho, a densidade de partículas

Tabela 2. Estimadores dos parâmetros da regressão para resistência mecânica à penetração (RP), em função da umidade volumétrica do solo $(\theta)$ e da densidade do solo $(\mathrm{DS})^{(1)}$.

\begin{tabular}{lccccc}
\hline Solo & $\mathrm{a}$ & $\mathrm{b}$ & $\mathrm{c}$ & Valor de $\mathrm{F}$ & Valor de $\mathrm{p}$ \\
\hline $\mathrm{L}_{480}$ & 0,0056 & 5,2581 & $-3,4878$ & 100,83 & $<0,001$ \\
$\mathrm{~N}_{520}$ & 0,0093 & 5,2722 & $-2,6047$ & 191,77 & $<0,001$ \\
$\mathrm{~L}_{640}$ & 0,0105 & 4,5746 & $-4,5593$ & 114,60 & $<0,001$ \\
\hline
\end{tabular}

${ }^{(1)} \mathrm{RP}=\mathrm{a} \times \mathrm{DS}^{\mathrm{b}} \times \theta^{\mathrm{c}}$, nos três solos $\left(\mathrm{n}=98\right.$ para $\mathrm{L}_{480}, \mathrm{~N}_{520}$ e $\left.\mathrm{L}_{640}\right) . \mathrm{L}_{480}$, Latossolo Vermelho distrófico; $\mathrm{N}_{520}$, Nitossolo Vermelho distroférrico; e $\mathrm{L}_{640}$, Latossolo Vermelho aluminoférrico. 

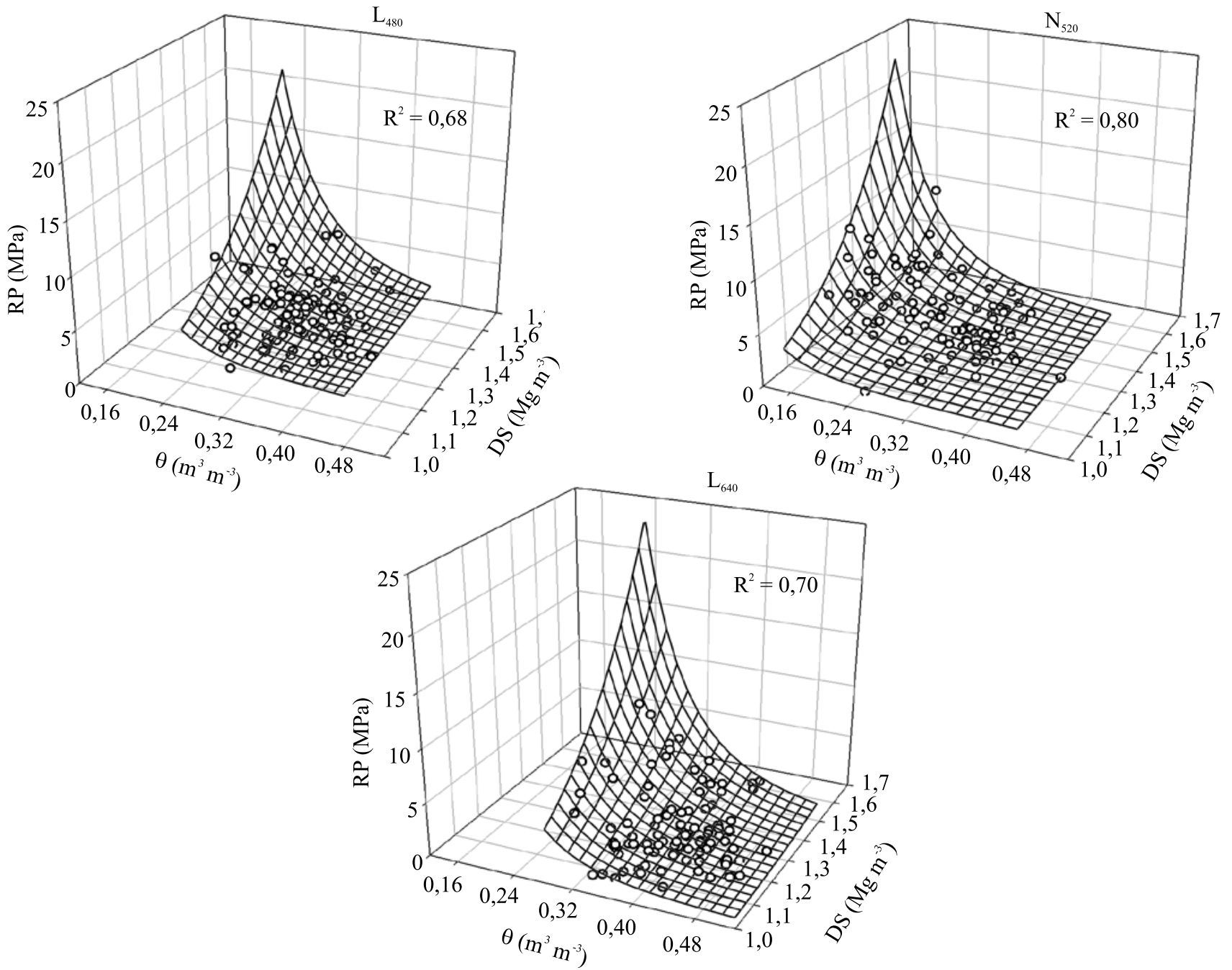

Figura 1. Resistência mecânica do solo à penetração (RP) em função da umidade do solo $(\theta)$ e da densidade do solo (DS). A função de Busscher (1990) foi ajustada aos dados com $\mathrm{n}=98$ para $\mathrm{L}_{480}, \mathrm{~N}_{520} \mathrm{e} \mathrm{L}_{640}$, após extração de outliers. $\mathrm{L}_{480}$, Latossolo Vermelho distrófico; $\mathrm{N}_{520}$, Nitossolo Vermelho distroférrico; e $\mathrm{L}_{640}$, Latossolo Vermelho aluminoférrico.
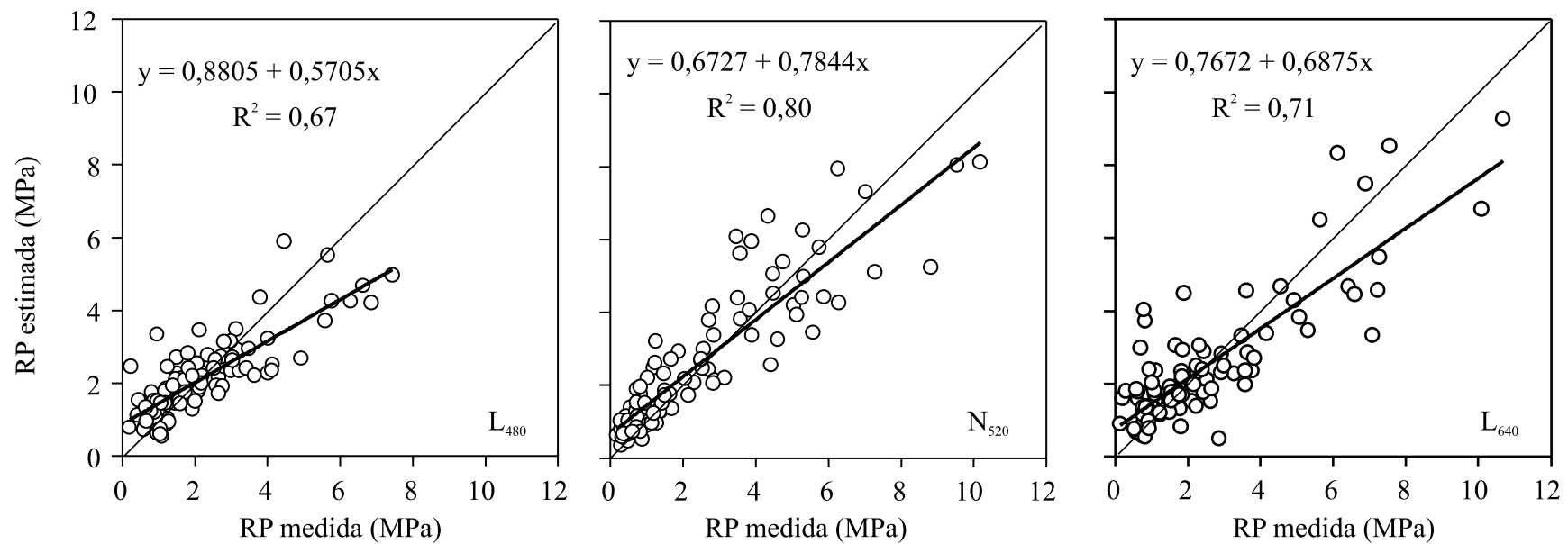

Figura 2. Ajuste da resistência mecânica à penetração (RP) estimada em função da RP medida para os três solos avaliados. $\mathrm{L}_{480}$, Latossolo Vermelho distrófico; $\mathrm{N}_{520}$, Nitossolo Vermelho distroférrico; e $\mathrm{L}_{640}$, Latossolo Vermelho aluminoférrico. 
do solo foi obtida, tendo-se obtido valores reais, para, então, determinar a porosidade de aeração, o que gerou variabilidade no ajuste desse limite. A densidade de partículas média foi de 2,50, 2,51 e 2,77 para $\mathrm{L}_{480}, \mathrm{~N}_{520}$ e $\mathrm{L}_{640}$, respectivamente, tendo diferido consideravelmente da utilizada como padrão $\left(2,65 \mathrm{~g} \mathrm{~cm}^{-3}\right)$. O reflexo na diferença da densidade de partículas na determinação da porosidade de aeração entre diferentes solos pode ser constatado na Figura $3 \mathrm{~B}$.
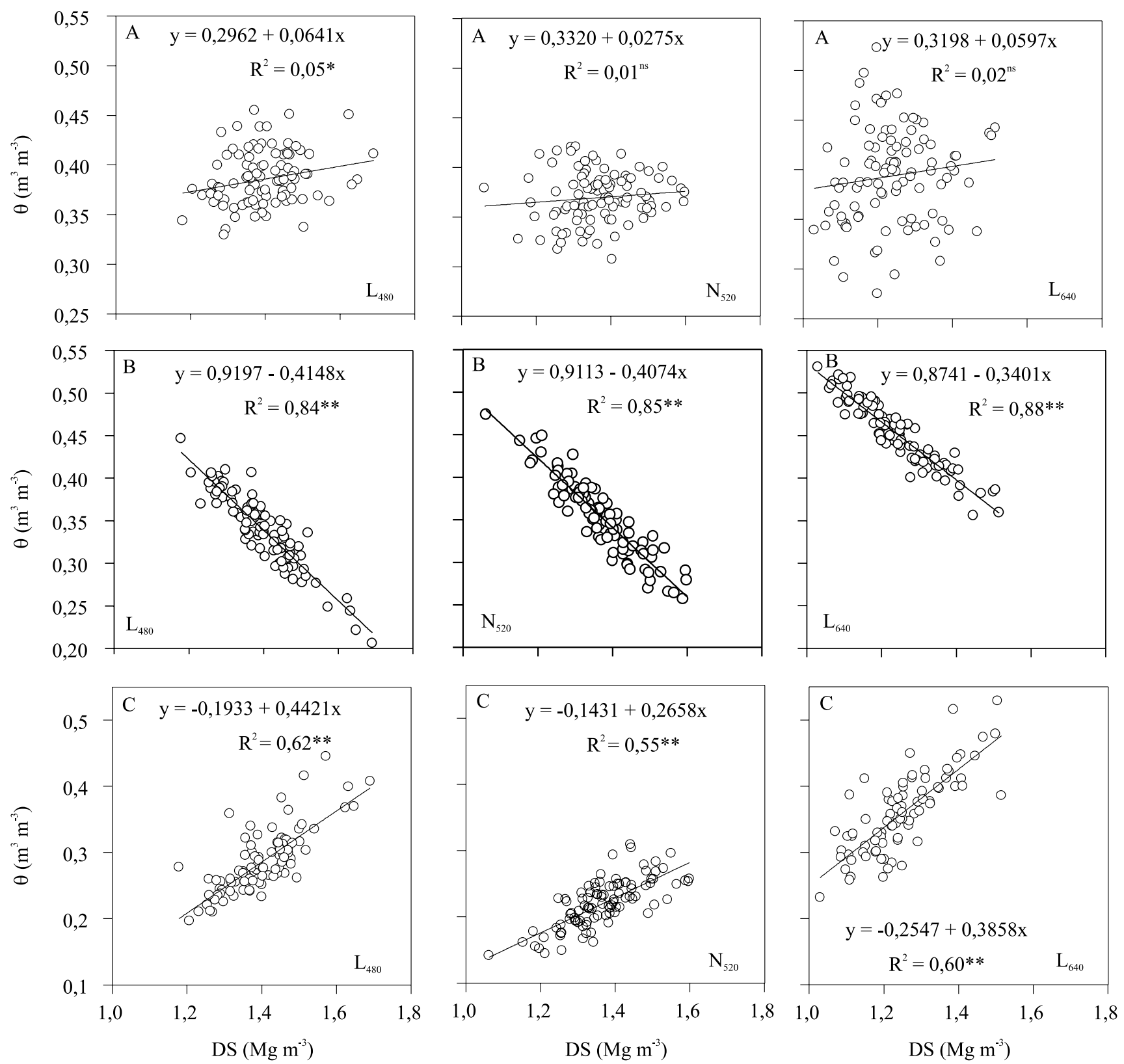

Figura 3. Ajuste da umidade volumétrica do solo $(\theta)$ para os limites do intervalo hídrico ótimo, em função da densidade do solo (DS). A, capacidade de campo ( $\psi=-0,006 \mathrm{MPa})$, com $\mathrm{n}=99$ para $\mathrm{L}_{480}$ e $\mathrm{N}_{520}$, e $\mathrm{n}=100$ para $\mathrm{L}_{640}$; $\mathrm{B}$, porosidade de aeração $\left(0,10 \mathrm{~m}^{3} \mathrm{~m}^{-3}\right)$, com $\mathrm{n}=99$ para $\mathrm{L}_{480}, \mathrm{~N}_{520}$ e $\mathrm{L}_{640}$; e $\mathrm{C}$, ponto de murcha permanente $(\psi=-1,5 \mathrm{MPa})$, com $\mathrm{n}=99$ para $\mathrm{L}_{480}, \mathrm{~N}_{520}$ e $\mathrm{L}_{640} .{ }^{*} \mathrm{e} * *$ Significativo a 5 e $1 \%$ de probabilidade, respectivamente. ${ }^{\text {ns }}$ Não significativo pelo teste $\mathrm{F}$, a $5 \%$ de probabilidade. $\mathrm{L}_{480}$, Latossolo Vermelho distrófico; $\mathrm{N}_{520}$, Nitossolo Vermelho distroférrico; e $\mathrm{L}_{640}$, Latossolo Vermelho aluminoférrico. 
A variação na DS determinou em 62,55 e $60 \%$ a variação na umidade no $\mathrm{PMP}$, para $\mathrm{L}_{480}, \mathrm{~N}_{520}$ e $\mathrm{L}_{640}$, respectivamente (Figura $3 \mathrm{C}$ ). Apesar de serem coeficientes de determinação altos, boa parte da variação na umidade no PMP não pode ser atribuída exclusivamente à DS, o que mostrou que esse limite do IHO é afetado por outros fatores.

O limite inferior do IHO foi a RP para os três solos avaliados (Figura $4 \mathrm{~A}$ ). $\mathrm{O}$ valor de $\mathrm{RP}=2 \mathrm{MPa}$ geralmente é utilizado na literatura como limitante ao desenvolvimento das plantas (Silva et al., 1994; Leao et al., 2005; Safadoust et al., 2014; Cecagno et al., 2016); porém, pode não representar limitações para culturas como a de soja, em solo mantido sob sistema plantio direto (Klein \& Camara, 2007). Dessa forma, o limite inferior do IHO depende muito do valor da RP crítica a ser assumida, assim como a RP ou o PMP ser o limite para a maior ou a menor parte das DS obtidas. $\mathrm{O}$ valor de RP a ser considerado como limitante ainda não foi definitivamente estabelecido e, portanto, pode tornar o IHO mais ou menos amplo.

A densidade do solo crítica (DSc), definida como a DS na qual o IHO é igual a zero (Leao et al., 2005), foi de $1,442,1,514$ e $1,246 \mathrm{Mg} \mathrm{m}^{-3}$ para $\mathrm{L}_{480}, \mathrm{~N}_{520} \mathrm{e}$ $\mathrm{L}_{640}$, respectivamente. A CC foi o limite superior até a DS de $1,302 \mathrm{Mg} \mathrm{m}^{-3}$, para $\mathrm{L}_{480}$, e de $1,332 \mathrm{Mg} \mathrm{m}^{-3}$ para $\mathrm{N}_{520}$ (Figura $4 \mathrm{~A}$ ). Em densidades superiores a essas, até a densidade do solo crítica (DSc), nestes dois solos, a porosidade de aeração substituiu a $\mathrm{CC}$ e foi o limite superior; porém, para $\mathrm{L}_{640}$, a $\mathrm{CC}$ continuou sendo o limite superior. Ao se considerar o número de amostras com DS inferior à DSc, a CC foi
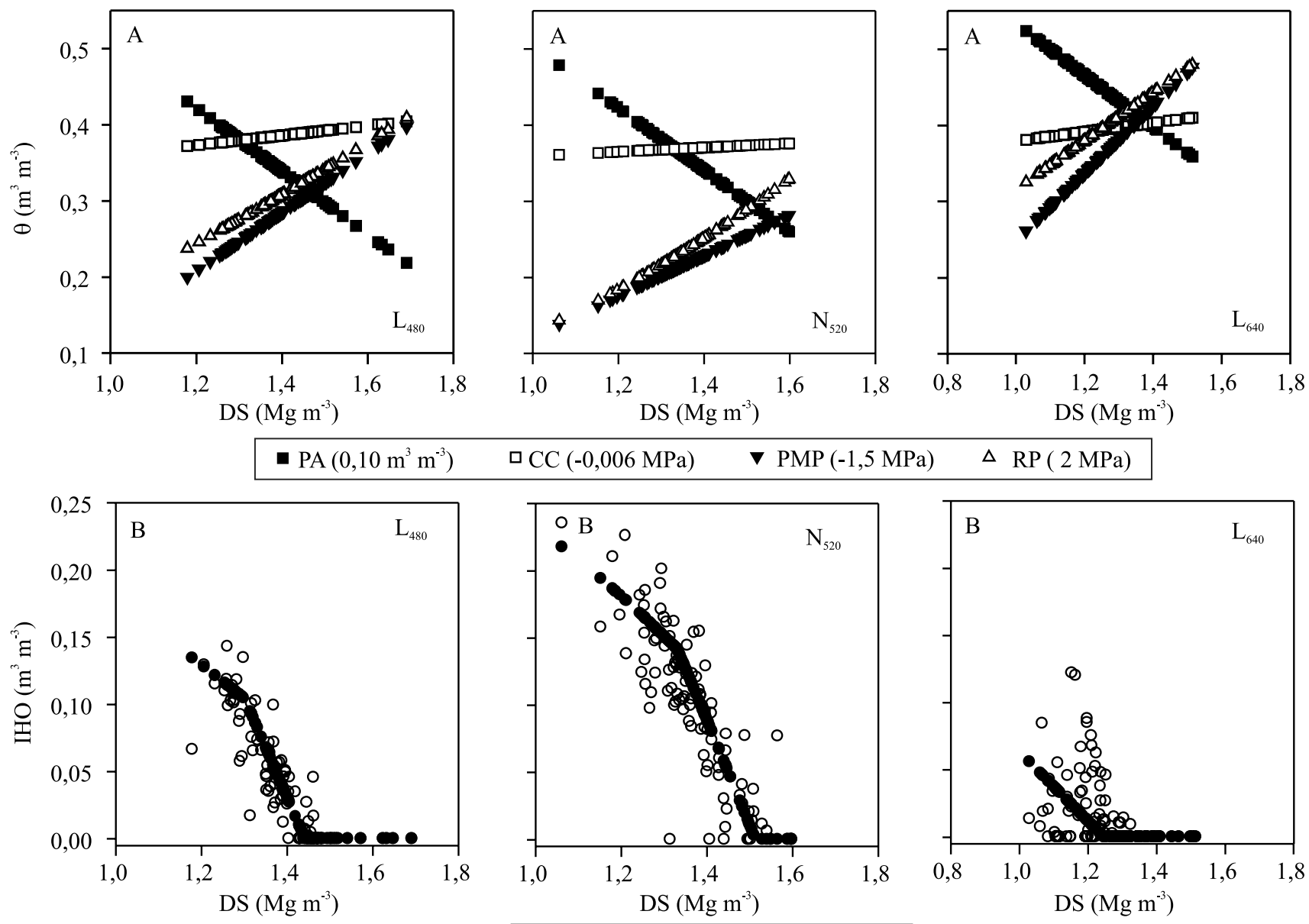

$\square \mathrm{CC}(-0,006 \mathrm{MPa}) \quad \nabla \mathrm{PMP}(-1,5 \mathrm{MPa}) \quad \Delta \mathrm{RP}(2 \mathrm{MPa})$
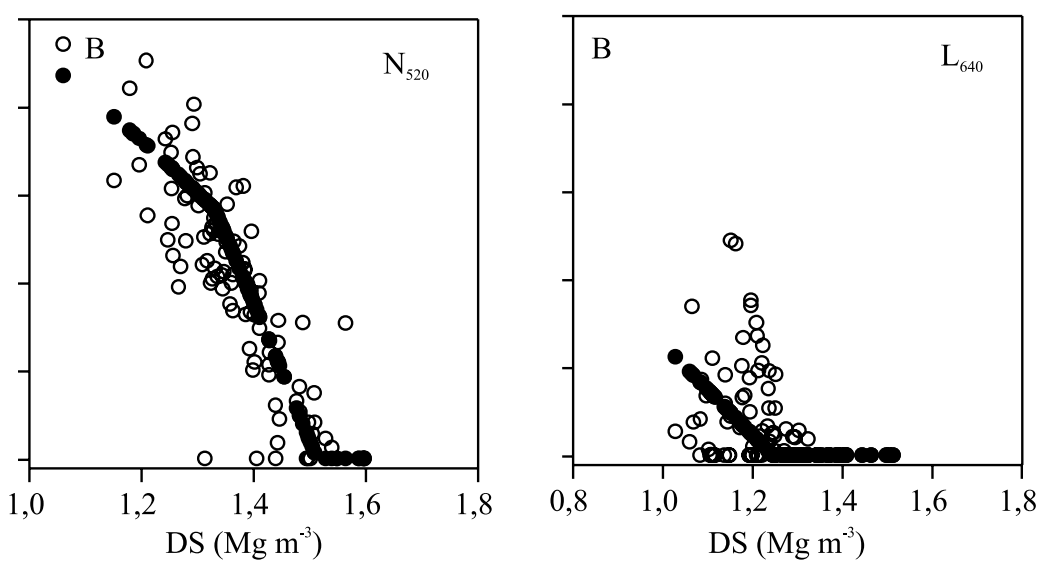

○ IHO Medido $\bullet$ IHO Estimado

Figura 4. Umidade volumétrica do solo $(\theta)$ para porosidade de aeração (PA), capacidade de campo (CC), ponto de murcha permanente (PMP) e resistência mecânica do solo à penetração (RP) crítica de $2 \mathrm{Mpa}(\mathrm{A})$, bem como intervalo hídrico ótimo (IHO) estimado e medido em função da densidade do solo (DS) (B). $\mathrm{L}_{480}$, Latossolo Vermelho distrófico; $\mathrm{N}_{520}$, Nitossolo Vermelho distroférrico; e $\mathrm{L}_{640}$, Latossolo Vermelho aluminoférrico. 
o limite superior em 28,1, 39,8 e 100\% das amostras, respectivamente, para $\mathrm{L}_{480}, \mathrm{~N}_{520}$ e $\mathrm{L}_{640}$. Todos os valores foram calculados a partir das funções dos limites do IHO. A CC representou um percentual considerável de amostras no limite superior, apesar dos baixos coeficientes de determinação (Figura $3 \mathrm{~A}$ ).

O IHO foi obtido como a diferença entre o menor valor de $\theta$, para as funções que delimitam o limite superior, e o maior valor de $\theta$, para as funções que delimitam o limite inferior (Figura $4 \mathrm{~B}$ ). Para essa determinação, foram considerados os valores estimados das regressões e os valores obtidos, à exceção da RP. Observaram-se discrepâncias entre os IHOs delimitados pelos valores estimados pelas regressões e os IHOs delimitados pelos valores reais obtidos; essas diferenças podem ser atribuídas aos desvios entre dados medidos e estimados.

Quanto maior o IHO, maior é a frequência e a probabilidade de que a umidade de um determinado solo esteja em uma condição não limitante (Keller et al., 2015). Entretanto, as regressões que definem os limites do IHO foram pouco explicativas a partir da variável independente DS. Os coeficientes de determinação para as regressões que definem a CC apresentaram valores baixos e, no caso dos solos $\mathrm{N}_{520}$ e $\mathrm{L}_{640}$, não significativos, mesmo com número elevado de amostras ( $n=99$ e $n=100$, respectivamente), o que prejudicou a confiabilidade das predições para o IHO (Figura 3 A). Outro fator a ser considerado é a variação nos valores de $\psi$ utilizados para representar a $\theta$ na $C$, ou seja, -0,006 MPa (Klein \& Camara, 2007; Blainski et al., 2009), -0,01 MPa (Silva et al., 1994; Leao et al., 2005) ou -0,033 MPa (Safadoust et al., 2014; Miola et al., 2015). A depender do $\psi$ a ser considerado, o IHO assume amplitude e DSc diferentes, o que dificulta sua utilização a partir destes critérios.

O IHO de solos agrícolas é utilizado para determinar a DSc, para que possam ser adotadas medidas para evitar que o solo atinja essa DS ou para fornecer embasamento teórico na tomada de decisão quando o solo se encontrar em densidades superiores. No entanto, quando ocorrem, ao mesmo tempo, IHOs iguais a zero e amplos em uma área aparentemente uniforme, com dimensões reduzidas, como as de 10x10 m, no caso dos três solos avaliados (Figura 5), a função e a viabilidade de uso desse indicador fica comprometida. Especialmente no solo $\mathrm{N}_{520}$, houve grande variação no IHO no espaço amostral, em um limiar de 0 a $0,20 \mathrm{~m}^{3} \mathrm{~m}^{-3}$.
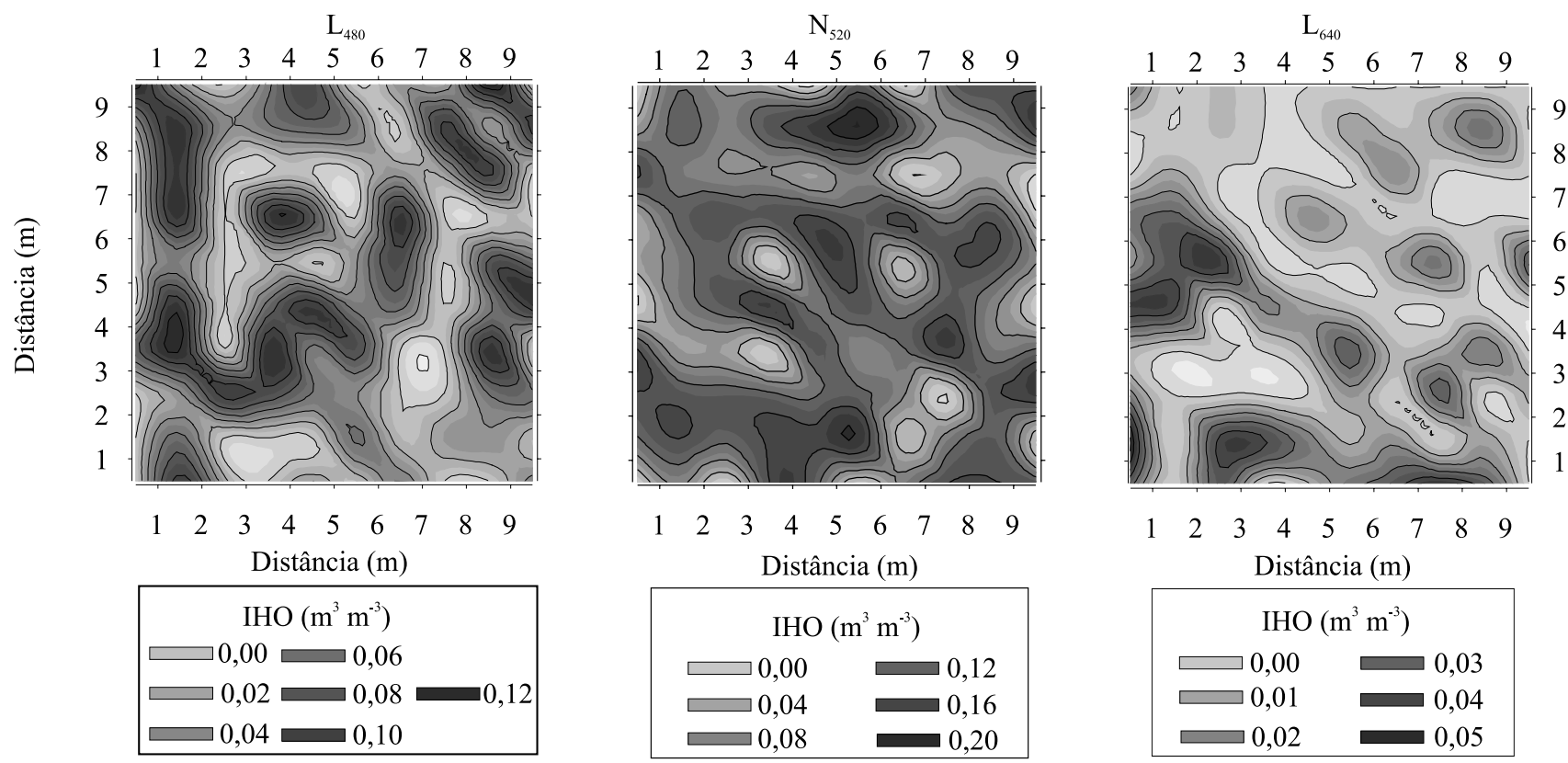

Figura 5. Mapa de contorno para o intervalo hídrico ótimo (IHO), em função do espaço amostral para os três solos avaliados $(\mathrm{n}=100)$ : $\mathrm{L}_{480}$, Latossolo Vermelho distrófico; $\mathrm{N}_{520}$, Nitossolo Vermelho distroférrico; e $\mathrm{L}_{640}$, Latossolo Vermelho aluminoférrico. 
Diversos trabalhos apontam para níveis de produtividade não condizentes com o IHO (Klein \& Camara, 2007; Gubiani et al., 2013a; Cecagno et al., 2016). Diante disso, a não ocorrência de correlação entre a produtividade de culturas e o IHO pode estar relacionada ao fato de as áreas apresentarem extremos de IHO, em razão da variabilidade espacial do solo (Figura 5).

\section{Conclusões}

1. As regressões que definem o limite da capacidade de campo do intervalo hídrico ótimo (IHO) não são acuradas em descrever a variação na umidade volumétrica do solo por meio da densidade do solo.

2. Os solos mantidos sob sistema plantio direto apresentam elevada variabilidade espacial para o IHO.

\section{Agradecimentos}

À Coordenação de Aperfeiçoamento de Pessoal de Nível Superior (Capes), à Fundação de Amparo à Pesquisa do Estado do Rio Grande do Sul (Fapergs) e ao Conselho Nacional de Desenvolvimento Científico e Tecnológico (CNPq), pelo apoio financeiro.

\section{Referências}

BETIOLI JÚNIOR, E.; MOREIRA, W.H.; TORMENA, C.A.; FERREIRA, C.J.B.; SILVA, A.P. da; GIAROLA, N.F.B. Intervalo hídrico ótimo e grau de compactação de um Latossolo Vermelho após 30 anos sob plantio direto. Revista Brasileira de Ciência do Solo, v.36, p.971-982, 2012. DOI: 10.1590/ S0100-06832012000300027.

BETZ， C.L.; ALLMARAS， R.R.; COPELAND, S.M.; RANDALL, G.W. Least limiting water range: traffic and long-term tillage influences in a Webster soil. Soil Science Society of America Journal, v.62, p.1384-1393, 1998. DOI: 10.2136/sssaj1998.03615995006200050034x.

BEUTLER, A.N.; CENTURION, J.F.; CENTURION, M.A.P. da C.; LEONEL, C.L.; SÃO JOÃO, A. de C.G.; FREDDI, O. da S. Intervalo hídrico ótimo no monitoramento da compactação e da qualidade física de um Latossolo Vermelho cultivado com soja. Revista Brasileira de Ciência do Solo, v.31, p.1223-1232, 2007. DOI: $10.1590 / \mathrm{S} 0100-06832007000600001$.

BLAINSKI, E.; GONÇALVES, A.C.A.; TORMENA, C.A.; FOLEGATTI, M.V.; GUIMARÃES, R.M.L. Intervalo hídrico ótimo num Nitossolo Vermelho distroférrico irrigado. Revista Brasileira de Ciência do Solo, v.33, p.273-281, 2009. DOI: 10.1590/S0100-06832009000200005.

BLAKE, G.R.; HARTGE, K.H. Bulk density. In: KLUTE. A. (Ed.). Methods of soil analysis: part 1: physical and mineralogical methods. $2^{\text {nd }}$ ed. Madison: American Society of Agronomy: Soil Science Society of America, 1986. p.363-375.

BUSSCHER, W.J. Adjustment of flat-tipped penetrometer resistance data to a common water content. Transactions of the American Society of Agricultural Engineering, v.33, p.519-524, 1990. DOI: $10.13031 / 2013.31360$.

CAMARGO, L.A.; MARQUES JÚNIOR, J.; PEREIRA, G.T. Spatial variability of physical attributes of an Alfisol under different hillslope curvatures. Revista Brasileira de Ciência do Solo, v.34, p.617-630, 2010. DOI: 10.1590/S0100-06832010000300003.

CAVAlCANTE, E.G.S.; ALVES, M.C.; SOUZA, Z.M. de; PEREIRA, G.T. Variabilidade espacial de atributos físicos do solo sob diferentes usos e manejos. Revista Brasileira de Engenharia Agrícola e Ambiental, v.15, p.237-243, 2011. DOI: 10.1590/ S1415-43662011000300003.

CECAGNO, D.; COSTA, S.E.V.G. de A.; ANGHINONI, I.; KUNRATH, T.R.; MARTINS, A.P.; REICHERT, J.M., GUBIANI, P.I.; BALERINI, F.; FINK, J.R.; CARVALHO, P.C. de F. Least limiting water range and soybean yield in a long-term, no-till, integrated crop-livestock system under different grazing intensities. Soil and Tillage Research, v.156, p.54-62, 2016. DOI: 10.1016/j.still.2015.10.005.

CLAESSEN, M.E.C. (Org.). Manual de métodos de análise de solo. 2.ed. rev. e atual. Rio de Janeiro: EMBRAPA-CNPS, 1997. 212p. (EMBRAPA-CNPS. Documentos, 1).

GUBIANI, P.I.; GOULART, R.Z.; REICHERT, J.M.; REINERT, D.J. Crescimento e produção de milho associados com o intervalo hídrico ótimo. Revista Brasileira de Ciência do Solo, v.37, p.1502-1511, 2013a. DOI: 10.1590/S0100-06832013000600007.

GUBIANI, P.I.; REICHERT, J.M.; REINERT, D.J. Indicadores hídrico-mecânicos de compactação do solo e crescimento de plantas. Revista Brasileira de Ciência do Solo, v.37, p.1-10, 2013b. DOI: 10.1590/S0100-06832013000100001.

GUEDES FILHO, O.; BLANCO-CANQUI, H.; SILVA, A.P. da. Least limiting water range of the soil seedbed for long-term tillage and cropping systems in the central Great Plains, USA. Geoderma, v.207-208, p.99-110, 2013. DOI: 10.1016/j.geoderma.2013.05.008.

GUIMARÃES, R.M.L.; TORMENA, C.A.; BLAINSKI, E.; FIDALSKI, J. Intervalo hídrico ótimo para avaliação da degradação física do solo. Revista Brasileira de Ciência do Solo, v.37, p.1512-1521,2013. DOI: 10.1590/S0100-06832013000600008.

HAIR JÚNIOR, J.F.; BLACK, W.C.; BABIN, B.J.; ANDERSON, R.E.; TATHAM, R.L. Análise multivariada de dados. 6.ed. Porto Alegre: Bookman, 2009. 688p.

JONG VAN LIER, Q. de; GUBIANI, P.I. Beyond the "Least Limiting Water Range": rethinking soil physics research in Brazil. Revista Brasileira de Ciência do Solo, v.39, p.925-939, 2015. DOI: $10.1590 / 01000683$ rbcs20140596.

KAISER, D.R.; REINERT, D.J.; REICHERT, J.M.; COLLARES, G.L.; KUNZ, M. Intervalo hídrico ótimo no perfil explorado pelas raízes de feijoeiro em um Latossolo sob diferentes níveis de compactação. Revista Brasileira de Ciência do Solo, v.33, p.845-855, 2009. DOI: 10.1590/S0100-06832009000400009. 
KELLER, T.; SILVA, A.P. da; TORMENA, C.A.; GIAROLA, N.F.B.; CAVALIERI, K.M.V.; STETTLER, M.; ARVIDSSON, J. SoilFlex-LLWR: linking a soil compaction model with the least limiting water range concept. Soil Use and Management, v.31, p.321-329, 2015. DOI: 10.1111/sum.12175.

KLEIN, V.A. Física do solo. 3.ed. Passo Fundo: UPF Editora, 2014. 263p.

KLEIN, V.A.; CAMARA, R.K. Rendimento da soja e intervalo hídrico ótimo em Latossolo Vermelho sob plantio direto escarificado. Revista Brasileira de Ciência do Solo, v.31, p.221-227, 2007. DOI: 10.1590/S0100-06832007000200004.

LAPEN, D.R.; TOPP, G.C.; GREGORICH, E.G.; CURNOE, W.E. Least limiting water range indicators of soil quality and corn production, eastern Ontario, Canada. Soil and Tillage Research, v.78, p.151-170, 2004. DOI: 10.1016/j.still.2004.02.004.

LEÃO, T.P.; SILVA, A.P da; PERFECT, E.; TORMENA, C.A. An algorithm for calculating the least limiting water range of soils. Agronomy Journal, v.97, p.1210-1215, 2005. DOI: 10.2134/ agronj2004.0229.

LEÃO, T.P.; SILVA, A.P. da; MACEDO, M.C.M.; IMHOFF, S.; EUCLIDES, V.P.B. Least limiting water range: a potential indicator of changes in near-surface soil physical quality after the conversion of Brazilian Savanna into pasture. Soil and Tillage Research, v.88, p.279-285, 2006. DOI: 10.1016/j.still.2005.06.014.

MIOLA, E.C.C.; PAULETTO, E.A.; LIMA, C.L.R. de; PINTO, L.F.S.; TIMM, L.C. Intervalo hídrico ótimo em solo construído após mineração de carvão em diferentes limites críticos de resistência à penetração e umidade. Revista Brasileira de Ciência do Solo, v.39, p.563-572, 2015. DOI: 10.1590/01000683rbcs20140184.

NEMÉNYI, M.; MESTERHÁZI, P.A.; MILICS, G. An application of tillage force mapping as a cropping management tool. Biosystems Engineering, v.94, p.351-357, 2006. DOI: 10.1016/j.biosystemseng.2006.04.005.

SAFADOUST, A.; FEIZEE, P.; MAHBOUBI, A.A.; GHARABAGHI, B.; MOSADDEGHI, M.R.; AHRENS, B. Least limiting water range as affected by soil texture and cropping system. Agricultural Water Management, v.136, p.34-41, 2014. DOI: 10.1016/j.agwat.2014.01.007.

SILVA, A.P. da; IMHOFF, S.; KAY, B. Plant response to mechanical resistance and air-filled porosity of soils under conventional and no-tillage system. Scientia Agricola, v.61, p.451-456, 2004. DOI: 10.1590/S0103-90162004000400016.

SILVA, A.P. da; KAY, B.D.; PERFECT, E. Characterization of the least limiting water range of soils. Soil Science Society of America Journal, v.58, p.1775-1781, 1994. DOI: 10.2136/ sssaj1994.03615995005800060028x.

SILVA, A.P.; KAY, B.D.; TORMENA, C.A.; IMHOFF, S. Least limiting water range of soils. In: LAL, R. (Ed.). Encyclopedia of soil science. $2^{\text {nd }}$ ed. New York: Taylor \& Francis, 2006. v.2, p.1026-1029.

STRECK, E.V.; KÄMPF, N.; DALMOLIN, R.S.D.; KLAMT, E.; NASCIMENTO, P.C. do; SCHNEIDER, P.; GIASSON, E.; PINTO, L.F.S. Solos do Rio Grande do Sul. 2.ed. rev. e ampl. Porto Alegre: UFRGS: EMATER/RS-ASCAR, 2008. 222p.

TORMENA, C.A.; SILVA, A.P. da; LIBARDI, P.L. Soil physical quality of a Brazilian Oxisol under two tillage systems using the least limiting water range approach. Soil Tillage Research, v.52, p.223-232, 1999. DOI: 10.1016/S0167-1987(99)00086-0.

WU, L.; FENG, G.; LETEY, J.; FERGUSON, L.; MITCHELL, J.; MCCULLONGH-SANDEN, B.; MARKEGARD, G. Soil management effects on the nonlimiting water range. Geoderma, v.114, p.401-414, 2003. DOI: 10.1016/S0016-7061(03)00052-1.

Recebido em 22 de janeiro de 2016 e aprovado em 17 de junho de 2016 